\title{
An all-inkjet printed flexible capacitor on a textile using a new poly(4-vinylphenol) dielectric ink for wearable applications
}

\author{
Yi Li, Russel Torah, Steve Beeby and John Tudor \\ School of Electronics and Computer Science, Southampton University \\ Southampton, UK \\ Email: \{yl1g08, rnt, spb, mjt\}@ecs.soton.ac.uk
}

\begin{abstract}
This paper reports a flexible capacitor for wearable applications which has been all-inkjet printed on a standard 65/35 polyester cotton textile using a new poly(4-vinylphenol) (PVP) dielectric material. Capacitors form the basis of a variety of sensors, such as for proximity and touch, as well as electronic circuits. This paper reports a general fabrication printing process to realize capacitors on textiles. The parallel plate capacitor design uses a combination of heat curable silver ink and a new UV curable dielectric ink based on PVP, printed on to the textile. This new inkjet printable dielectric ink is ultra-violet (UV) cured for 100 seconds to achieve a thin dielectric film suitable for a textile based capacitor. After printing, the dielectric properties were measured and the cross-sectional film structure was observed using an SEM. The inkjet printed PVP film on the textile exhibited good insulating behavior and similar flexibility to the original textile.
\end{abstract}

\section{INTRODUCTION}

Smart fabrics have attracted growing interest in the last ten years, especially for wearable system applications [1]. An essential electronic component, necessary for wearable electronic circuits and sensors, is a flexible capacitor. An allinkjet printed capacitor is of great interest because of its use in direct write processed electronic sensors and circuits. Inkjet printing technology provides a potentially straightforward low-cost, non-contact solution to realise electronic circuits and sensors on flexible textile substrates in digitally defined patterns. The development of all-inkjet printing processes, curable at a sufficiently low temperature $\left(<150{ }^{\circ} \mathrm{C}\right)$ to avoid damage to the fabric substrate, has a critical role in achieving advanced inkjet printed wearable systems.

Inkjet printing is a non-contact additive technique enabling the deposition of electronic, functional and structural materials (e.g. conductor, insulator and semiconductor). Deposited patterns directly follow a pre-designed computer layout without utilising masks or subsequent etching processes. After ink deposition onto the substrate, a further curing treatment process, such as thermal or UV curing is required. Inkjet printing is a versatile prototyping tool with the ability to pattern diverse two and quasi three-dimensional shapes and devices (e.g. Micro ElectroMechanical Systems (MEMS) [2].
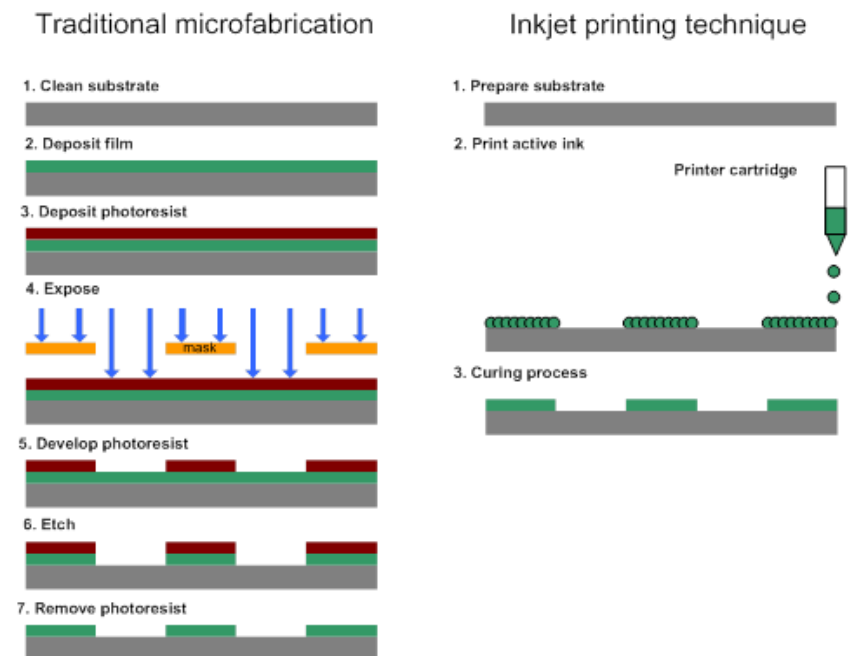

Figure 1. A comparison of the processing steps involved in substractive microfabrication and addictive direct writing printing.

In comparison to conventional microfabrication techniques, which utilise subtractive processes such as etching, inkjet printing significantly reduces the number of process steps, energy demanded and waste generated, as illustrated in Fig. 1. The term 'all-inkjet' printing is used when every layer of a complete device is patterned by inkjet printing without any other deposition methods. Using a single deposition method is clearly attractive from a production viewpoint. This paper reports the all-inkjet printing of a capacitor on a textile substrate for future use in wearable textile electronics/sensors.

\section{BACKGROUND}

There have been a number of approaches to capacitor fabrication using inkjet printing. Some use additional fabrication methods other than inkjet printing to realise the capacitor [3], others are based on rigid substrates [4, 5] or require temperatures $\left(>280{ }^{\circ} \mathrm{C}\right)[5]$ which are too high for the majority of fabrics. Reference [3] describes a capacitor in which the conductive plates are inkjet printed using a Poly (3, 4-ethylenedioxy thiophene)-poly

(styrenesulfonate) 
(PEDOT/PSS) conductive polymer ink on two flexible PET substrates. An ethyl-2-cyanoacrylate adhesive was used to stick the two PEDOT/PSS coated PET substrates together and act as the dielectric of the capacitor. Reference [4] describes an all polymer capacitor fabricated on a rigid substrate by allinkjet printing using PEDOT/PSS ink and a polyimide (amic acid solution) dielectric ink. In 2011 [5], an all-inkjet printed capacitor was fabricated on a rigid alumina substrate using a silver conductor ink cured at $370{ }^{\circ} \mathrm{C}$ and a $\mathrm{BaTiO}_{3}$ dielectric ink cured at $280{ }^{\circ} \mathrm{C}$.

For printing on fabric, it is essential to use inks with low curing temperatures, $100-150{ }^{\circ} \mathrm{C}$ depending on the fabric, to avoid damaging the fabric. In the case of an all-inkjet printed capacitor, the thickness, smoothness and dielectric properties of the printed dielectric will significantly affect its performance. UV-curing inkjet inks (e.g. acrylates and SU-8) have been shown to produce a thick dielectric layer, around 7 $\mu \mathrm{m}$ per print layer, after curing [6]. Typically, two to three inkjet printed dielectric layers are required to avoid pinholes in the dielectric film which will cause short circuits or reduce the performance of the dielectric. A thicker inkjet printed layer will decrease the capacitance of the capacitor but also precludes the use of the dielectric layer in other all-inkjet printed applications such as printed transistors. Solvent based inkjet printable dielectric inks can produce a thin dielectric layer, but these typically require higher curing temperatures (e.g. a heat curable inkjet PVP layer requires $200{ }^{\circ} \mathrm{C}$ [7]). Therefore, an inkjet printable UV-cure dielectric ink for thin films at room temperature is of significant interest.

\section{MATERIALS AND FABRICATION}

\section{A. Materials}

A standard $65 / 35$ polyester/cotton textile (supplied by Klopman Ltd.) is used as the flexible textile substrate in this work, as it is the most commonly used textile for clothing. The fabric was pre-treated with screen printed polyurethane (Fabink-UV-IF1 supplied by Smart Fabric Inks Ltd.) as an interface layer. The interface layer is designed to reduce the original textile surface roughness significantly down to a few microns as shown in Fig. 2 [8]. The thermal-cure silver inkjet printable conductive ink (U5714) is supplied from SunChemical Ltd. The UV-cure PVP inkjet printable dielectric ink is prepared from a mixture of PVP and poly(melamine-co-formaldehyde) methylated solution (PMF) in 1-hexanol with additional polymerization initiator (PI) bis(4-tert-butylphenyl)iodonium triflate (DtBPIT). PMF is used as the cross linking agent to polymerise the PVP monomers into a chain while the PI is used as the polymerization initiator to catalyse the polymerization between the PVP and PMF.

\section{B. Inkjet printing}

We used a Dimatix DMP-2831 inkjet printer with a piezoelectric cartridge of $1.5 \mathrm{~mL}$ capacity and a $10 \mathrm{pL}$ drop volume. Printable inks have a narrow acceptable range of rheological properties so that droplets fire continuously in the required landing location. An ideal ink for the DMP 2831 is a stable suspension with low evaporation, viscosity of 10 to 12 $\mathrm{mPa} . \mathrm{s}$ and surface tension of 0.028 to $0.033 \mathrm{~N} / \mathrm{m}$. Printed

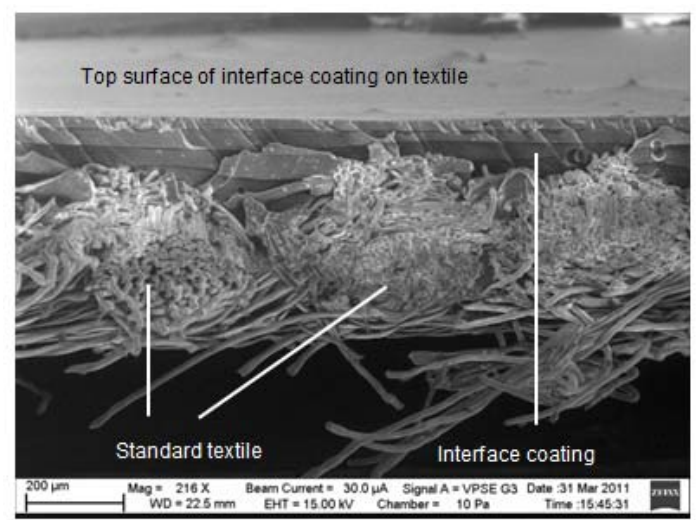

Figure 2. A SEM cross sectional image of interface layer coated standard $65 / 35$ polyester/cotton textile.

pattern resolution is controlled by adjusting the droplet spacing between $5 \mu \mathrm{m}$ and $254 \mu \mathrm{m}$ for the DMP-2831. If the droplet spacing is too low the volume of printed ink will be too high per unit area which results in pattern bleeding [6].

\section{Device fabrication}

The inkjet printed capacitor layout is shown in Fig. 3 (a) and was designed using Tanner L-Edit software. The first inkjet printed layer is the conductive silver bottom electrode, represented in blue. The second inkjet printed layer is the dielectric PVP layer, represented in green. The third inkjet printed layer is the conductive silver top electrode, represented in red. Alignment marks on the top left and bottom right of the layout are to ensure the two parallel plate capacitor electrodes are precisely aligned. To achieve a high quality capacitor, all three layers need low surface roughness and good uniformity. It is important to eliminate pinholes and avoid missing or poorly printed lines in the dielectric layer. Therefore, a large dielectric pattern is designed to ensure the ink is firing consistently before reaching the active dielectric layer and also avoid short circuits. This avoids the problem, common with inkjet printing, that the first few drops of each line can print unreliably and cause possible misalignment between multi layers.

The first conductive electrode was inkjet printed on the pre-treated textile substrate with $15 \mu \mathrm{m}$ droplet spacing at $21^{\circ} \mathrm{C}$. The nozzle diameter for the DMP-2831 $(\sim 20 \mu \mathrm{m})$ produces a droplet of $60 \mu \mathrm{m}$ diameter. Choosing a $30 \mu \mathrm{m}$ drop spacing improves the conductivity since the drops overlap but results in strongly castellated edges to the lines. A $15 \mu \mathrm{m}$ drop spacing provides good conductivity and line edge definition combined with acceptable ink usage. The conductive silver

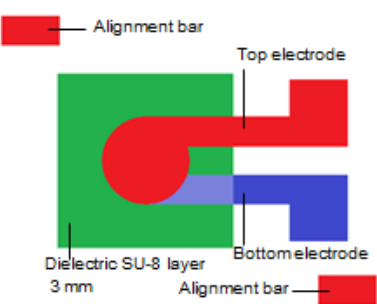

(a)

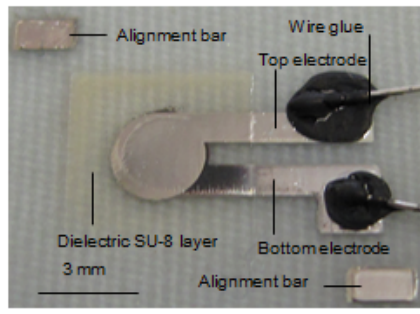

(b)
Figure 3. Top views of (a) the L-Edit design for the inkjet printed capacitor and (b) of the all inkjet printed capacitor with wires attached. 
ink used in this work does not require pre-treatment of the interface layer as the wettability is good. Once the bottom silver conductor is printed, it is cured at $150^{\circ} \mathrm{C}$ for 10 minutes in a conventional oven. $150{ }^{\circ} \mathrm{C}$ was chosen because it provides a suitable compromise between sufficient conductivity and compatibility with fabrics. Increasing this temperature will improve the conductivity but will damage the fabric. Next, the UV-cure PVP dielectric layer was printed. This was printed with the same $15 \mu \mathrm{m}$ droplet spacing at $21^{\circ} \mathrm{C}$. Choosing the same drop spacing enhances alignment between layers. Two dielectric layers were inkjet printed to minimise defects due to pinholes or missing lines. The dielectric layer was then crosslinked in a UV chamber (Mercury bulb, $160 \mathrm{~nm}$ wavelength transmission) for $100 \mathrm{~s}$. Finally the top silver electrode was printed and cured as before. Wires were connected to the printed electrodes using a conductive adhesive (AP Anders Products). The final printed capacitor is shown in Fig. 3 (b).

\section{RESUlTS AND DICUSSION}

\section{A. Film characterisation}

Both cured silver and PVP layers exhibited good chemical resistance to most commonly used solvents, for example, ethanol (U5714 ink's solvent) and 1,2-dichlorobenzene (polythiophene's solvent). In addition, depositing the UV-cure PVP ink did not change the textile interface layer's chemical or physical properties. All three layers showed good smoothness across the effective area of the capacitor. The thicknesses of the three functional layers were measured in cross section using a scanning electron microscope (SEM) as shown in Fig. 4 (a). The UV cured PVP layer thickness was $4.5 \mu \mathrm{m}$ and the silver thickness was around 4-5 $\mu \mathrm{m}$. Fig. 4 (b) shows that there is a random series of $100-300 \mathrm{~nm}$ diameter air bubbles in the inkjet printed UV cured PVP layer. These nanometer sized air bubbles will adversely affect the dielectric properties of the PVP layer.

\section{B. Capacitor measurement}

Two inkjet printed capacitors and a commercial $150 \mathrm{pF}$ capacitor were evaluated using a Wayne Kerr 6500B precision impedance analyser. The blue line in each graph in Fig. 5 is

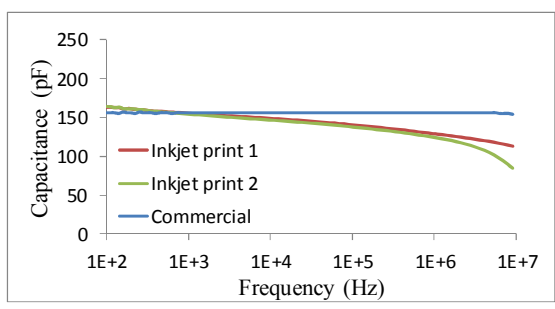

(a)

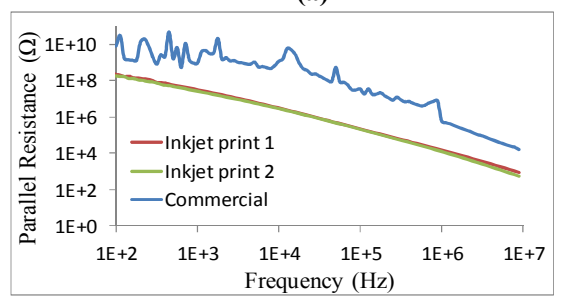

(c)

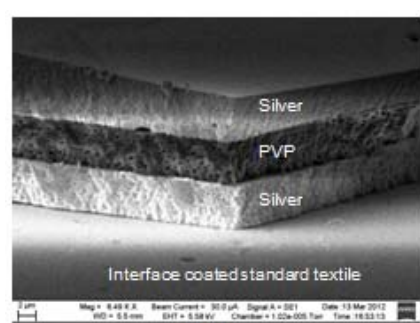

(a)

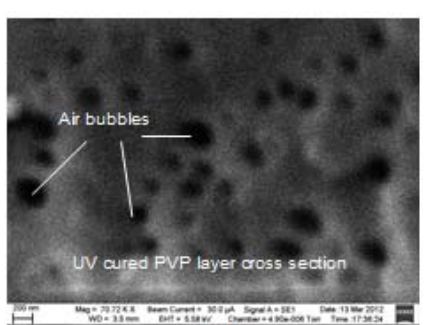

(b)
Figure 4. SEM cross sectional images for (a) an all-inkjet printed capacitor on textile with U5714 silver ink and new UV-cure PVP ink and (b) a close up of the PVP layer showing nanometer sized air bubbles.

the commercial $150 \mathrm{pF}$ capacitor. The capacitance of the printed samples varies from $163 \mathrm{pF}$ at $100 \mathrm{~Hz}$ to $113 \mathrm{pF}$ at 9 $\mathrm{MHz}$, a reduction of around $29 \%$, as shown in Fig. 5 (a). The printed ones' capacitance values start to decrease after $10 \mathrm{kHz}$. It may be caused by the high dielectric loss tangent, which means there is a large dielectric absorption causing a more pronounced deterioration of capacitance with frequency. Equation (1) [9] defines the capacitance as a function of frequency.

$$
C_{f}=C_{0} \times\left(j \times f / f_{0}\right)^{k}
$$

Where $C_{f}$ is the value of the capacitance at a frequency $f$, $C_{0}$ is the value at frequency $f_{0}, k$ is a negative constant that sets the capacitance change per decade of frequency. Based on (1), where $C_{0}$ is the capacitance measured at low frequency. There are two terms extracted from $\left(j \times f / f_{0}\right)^{k}$ : the pure phase part $j^{k}$ and the magnitude part $\left(f / f_{0}\right)^{k}$. As the frequency increases, the term $\left(f / f_{0}\right)^{k}$ decreases. The magnitude is determined by the dielectric loss tangent which decreases inversely proportionally to the frequency [9]. The capacitor area is $7.07 \mathrm{~mm}^{2}$ based on a designed diameter of 3 $\mathrm{mm}$. The measured thickness of the cross linked PVP dielectric layer is $4.5 \mu \mathrm{m}$, as shown in Fig. 4 (a) and capacitance is $163 \mathrm{pF}$ at $100 \mathrm{~Hz}$. Therefore the relative permittivity of inkjet printed PVP layer can be calculated to be 11.7 , as it is almost doubled the previously reported solution processed PVP relative permittivity of 6.4 [10]. For the

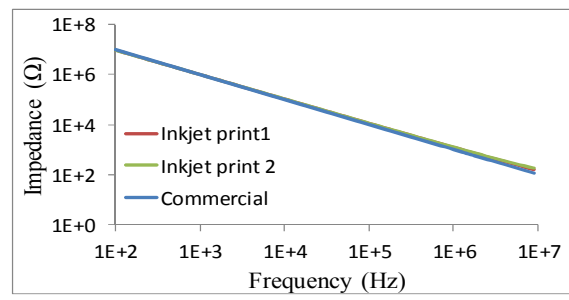

(b)

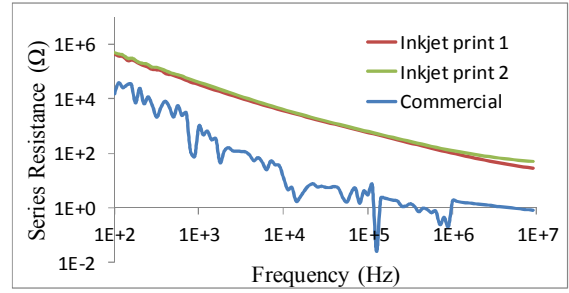

(d)

Figure 5. Graphs for (a) Capacitance, (b) Impedance, (c) Parallel resistance and (d) Series resistance as a function of frequency for two inkjet printed and a 150 $\mathrm{pF}$ commercial capacitor. 
commercial $150 \mathrm{pF}$ ceramic capacitor (TruCap $150 \mathrm{P} 50 \mathrm{~V} 10 \%$ ), there is no reduction in capacitance up to a frequency of $9 \mathrm{MHz}$. Fig. 5 (b) compares the impedance change between the three devices. The results show that there is good correlation between the three measured results.

\section{Results discussion}

An ideal capacitor has infinite parallel and zero series resistance. In practice, capacitors have imperfections which create parallel and series resistances. A finite parallel resistance can occur from conductivity of the dielectric causing a leakage current between the electrodes. Ultimately, the capacitor will be discharged by the parallel resistance with a discharge time controlled by its value. The equivalent series resistance is determined by the resistance of the electrodes and the loss tangent of the dielectric.

Fig. 5 (c) shows the change in parallel resistance as a function of frequency. The two inkjet printed capacitors' resistance values are very similar, while that of the commercial capacitor is around 1 to 2 magnitude higher. However, the three measured results showed the same general trend in the plotted graph. In contrast, the parallel resistance of the inkjet printed capacitor is lower than the commercial one as a function of frequency resulting in a higher leakage current. This higher leakage current could be because the inkjet printed layer is less uniform than the ceramic capacitor as a result of the nanometer sized air bubbles in the cured PVP layers which will cause variations in the dielectric properties.

Fig. 5 (d) shows the series resistances over the same frequency range. The two inkjet printed capacitors' resistances are very similar whilst the commercial capacitor's series resistance is around 1 to 2 magnitude lower. However, the three measured results follow the same trend with the series resistance of the commercial capacitor decreasing at a slightly higher rate as a function of frequency. This higher series resistance is partly due to the higher dielectric loss but the results from Fig. 5 (a) show that the capacitance did not change significantly compared to the commercial capacitor so therefore the dielectric loss is reasonably acceptable. Therefore, another possible cause of the high series resistance will be the lower conductivity of the printed tracks compared to the nickel/copper wire used in the ceramic capacitors.

\section{CONCLUSIONS}

An all inkjet printed parallel plate flexible capacitor has been printed on pre-treated standard 65/35 textile. A low temperature process $\left(150{ }^{\circ} \mathrm{C}\right.$ for 10 minutes $)$ has been presented. The device is constructed from silver ink and a new UV-cure PVP dielectric ink. The key electrical parameters of the two inkjet printed capacitors have been measured and compared to a $150 \mathrm{pF}$ commercial ceramic capacitor. The initial values of the two all inkjet printed capacitors' capacitance, impedance, parallel resistance and series resistance are very similar to each other. However, the results show that the dielectric loss, leakage current and plate resistances are slightly higher for the inkjet printed capacitor. These performance parameters could be improved with more uniformly printed layers to reduce the occurrences of pinholes in the printed layers.

Nanometer sized air bubbles have been observed in the dielectric layer which will affect the dielectric properties. Future work will attempt to reduce these air bubbles by adding a pre-soft bake in the curing process to remove the 1-hexanol solvent before cross-linking in the UV chamber. Having proven the functionality of the all inkjet printed capacitor on the pre-treated standard $65 / 35$ polyester cotton textile, future work will increase the capacitor's performance by further investigating the new UV-cure PVP dielectric ink's formulation and curing process. These results show that the UV-cure PVP ink is a promising option for future inkjet printable dielectric ink for use in inkjet printed wearable sensor fabrication.

\section{ACKNOWLEDGEMENTS}

This work has been supported by the EPSRC Fellowship grant number EP/I005323/1 (spb) and by the EU under the FP7 project MICROFLEX grant number CP-IP 211335-2 (rnt, mjt).

\section{REFERENCES}

[1] Rienzo, M. D., Rizzo, F., Meriggi, P., Bordoni, B., Brambilla, G., Ferratini, M. and Castiglioni, P., (2006), "Applications of a textilebased wearable system for vital signs monitoring", Engineering in Medicine and Biology Socirty, Proceedings of the 28th IEEE EMBS Annual International Conference, pp. 2223-2226.

[2] S. B. Fuller, E. J. Wilhelm, J. M. Jacobson, "Ink-jet printed nanoparticle microelectromechanical systems", Journal of Microelectromechanical Systems, 2002, vol. 11, number 1, pp. 54-60.

[3] C. Sriprachuabwong, C. Srichan, T. Lomas, A. Tuantranont, "Simple RC low pass filter circuit fabricated by unmodified desktop inkjet printer", Electrical Engineering and Electronics Computer .

[4] Y. Liu, T. Cui, K. Varahramyan, "All-polymer capacitor fabricated with inkjet printing technique", Solid-State Electronics, 2003, vol. 47, pp. 1543-1548.

[5] J. Lim, J. Kim, Y. J. Yoon, H. Kim, H. G. Yoon, S. Lee, J. Kim, “'Allinkjet-printed metal-insulator-metal (MIM) capacitor", Current Applied Physics, 2011, article in press.

[6] Y. Li, R. Torah, S. Beeby and J. Tudor, "An all-inkjet printed flexible capacitor for wearable applications", Design, Test, Integration \& Packaging of MEMS/MOEMS Symposium, April 2012, pp. 192-195.

[7] M. Hwang, H. Lee, Y. Jang, J. Cho, S. Lee, D. Kim, K. Cho, Macromol. Res. vol. 17, No. 6, 2009, pp.436-440.

[8] Y. Li, R. Torah, S. Beeby and J. Tudor, “'Inkjet printed flexible antenna on textile for wearable applications", the $88^{\text {th }}$ Textile Institute World Conference, May 2012.

[9] H. Johnson, Dielectric loss tangents, High Speed Digital Design Online Newsletter, Vol. 4, Issue 5.

[10] Z. Bao and J. Locklin, 'Organic Field-effect Transistors', Chapter 3.2, pp. 237, ISBN 0-8493-8080-4. 